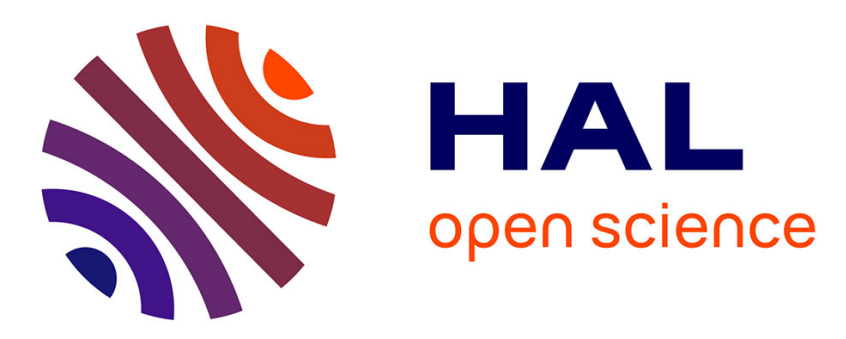

\title{
Hybrid 2D-3D ultrasound registration for navigated prostate biopsy
}

Sonia-Yuki Selmi, Emmanuel Promayon, Jocelyne Troccaz

\section{To cite this version:}

Sonia-Yuki Selmi, Emmanuel Promayon, Jocelyne Troccaz. Hybrid 2D-3D ultrasound registration for navigated prostate biopsy. International Journal of Computer Assisted Radiology and Surgery, 2018, 13 (7), pp.987-995. 10.1007/s11548-018-1736-4 . hal-01739427

\section{HAL Id: hal-01739427 \\ https://hal.science/hal-01739427}

Submitted on 21 Mar 2018

HAL is a multi-disciplinary open access archive for the deposit and dissemination of scientific research documents, whether they are published or not. The documents may come from teaching and research institutions in France or abroad, or from public or private research centers.
L'archive ouverte pluridisciplinaire HAL, est destinée au dépôt et à la diffusion de documents scientifiques de niveau recherche, publiés ou non, émanant des établissements d'enseignement et de recherche français ou étrangers, des laboratoires publics ou privés. 
Noname manuscript No.

(will be inserted by the editor)

\title{
Hybrid 2D-3D Ultrasound Registration For Navigated Prostate Biopsy
}

\author{
Sonia-Yuki Selmi · Emmanuel \\ Promayon • Jocelyne Troccaz
}

Received: date / Accepted: date

\begin{abstract}
Purpose: We present a hybrid 2D-3D ultrasound (US) rigid registration method for navigated prostate biopsy that enables continuous localization of the biopsy trajectory during the exam.

Methods: Current clinical computer-assisted biopsy systems use either sensorbased or image-based approaches. We combine the advantages of both in order to obtain an accurate and real-time navigation based only on an approximate localization of the US probe. Starting with features extracted in both 2D and $3 \mathrm{D}$ images, our method introduces a variant of the Iterative Closest Point (ICP) algorithm. Among other differences to ICP, a combination of both the euclidean distance of feature positions and the similarity distance of feature descriptors is used to find matches between 2D and 3D features. The evaluation of the method is twofold. First, an analysis of variance on input parameters is conducted to estimate the sensitivity of our method to their initialization. Secondly, for a selected set of their values, the Target Registration Error (TRE) was calculated on 29,760 (resp. 4,000) registrations in two different experiments. It was obtained using manually identified anatomical fiducials.

Results: For 160 US volumes, from 20 patients, recorded during routine biopsy procedures performed in two hospitals by 6 operators, the mean TRE was $3.91 \pm 3.22 \mathrm{~mm}$ (resp. $4.37 \pm 2.62 \mathrm{~mm}$ ).

Conclusion: This work allows envisioning further developments for prostate navigation and their clinical transfer.
\end{abstract}

Keywords 2D-3D registration · Prostate biopsy · Ultrasound

We thank Dr P. Mozer (APHP) and Dr G. Fiard (CHUGA) for patient data. This work was supported by the French Industry Minister through the FUI program, MIRAS project, and the Investissements d'Avenir programme (Labex CAMI) under reference ANR-11-LABX0004 .

All authors at:

Univ. Grenoble Alpes, CNRS, CHU Grenoble Alpes, Grenoble INP, TIMC-IMAG, F-38000

Grenoble, France

E-mail: firstName.lastName@univ-grenoble-alpes.fr 


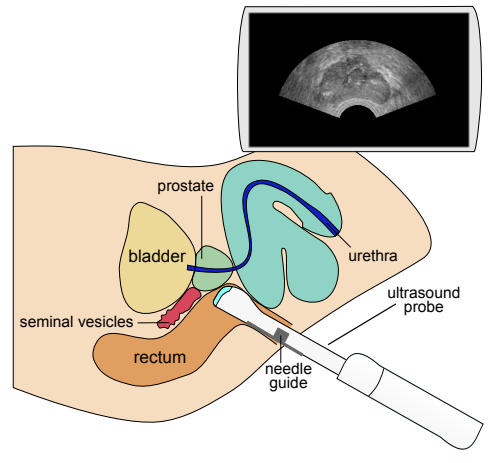

Fig. 1 TRUS-guided procedure

\section{Introduction}

Prostate biopsy is the confirmatory examination for diagnosis of prostate cancer, one of the most common cancer world-wide. Biopsy procedures are performed for histological analysis of tissue samples of the gland. Clinicians guide needles through patient's rectal wall using live 2D ultrasound (US) information acquired using a TransRectal US (TRUS) probe (see Fig.1). Random sampling (typically with 12-cores) and/or targeted sampling (when suspicious regions are detected in a Magnetic Resonance Imaging exam) are taken for further diagnosis. Random samples should be as evenly distributed as possible in the gland to maximize the probability of cancer detection if any. Moreover, knowing precisely sample locations may be crucial for reliable cancer diagnosis and appropriate treatment decision. This may also allow to envision the development of focal treatments.

However, this procedure is made difficult for several reasons: the biopsy is a 3D gesture while the provided information is bi-dimensional, the prostate is quite a symmetric organ, the US images are of low quality and present a lack of intrinsic contrast between tumor and non-tumor. As the prostate may be deformed and displaced during the procedure, current clinical techniques result in inaccurate biopsy sample location. This leads to a low correlation between the theoretical biopsy core locations and the histologic maps of the prostatectomy specimen [1].

To locate the exact position of the samples and to guide the clinician accurately towards a target, two different approaches were implemented in commercially available guidance systems proposed to clinicians: the sensor-based approach and the organ-based approach. The sensor-based approach involves a preoperative 3D-3D MRI to US registration just before the procedure. The US probe is then tracked during the procedure using an electromagnetic tracker [2] or mechanical arm [3] in order to update of the needle positioning. The organbased approach also involves a preoperative 3D/3D MRI to US registration just before the procedure. The prostate is then localized in the images for each biopsy using 3D-3D US to US registration [4]. In $[2,3] 3 \mathrm{D}$ pre-operative 
information is reconstructed from a set of localized 2D images whilst, in [4] a $3 \mathrm{D}$ US probe directly provides $3 \mathrm{D}$ intra-operative information. In both cases, the biopsy needle is inserted in a mechanical guide attached to the US probe and the probe position defines the potential needle trajectory in a fix external reference frame. The sensor-based real-time solution may lack accuracy because it can not take into account prostate and patient motions when the needle trajectory is updated. The organ-based solution demonstrates better accuracy but the update of the needle trajectory with respect to the prostate cannot be performed in real-time due to the $3 \mathrm{D}$ volume acquisition time and the non-rigid registration computation time.

2D-3D US registration for prostate biopsy navigation potentially offers an interesting compromise between fast computing time and accuracy. However using it for real-time navigation is very challenging due to (1) limited information from the $2 \mathrm{D}$ image about off-plane displacement and deformation of the prostate, (2) US imaging shortcomings (noise, artifact shadows and US signal attenuation with depth) and (3) the need for short computation time. Based on their previous work [3], De Silva et al. [5] describes 2D-3D rigid registration of prostate images using Normalized Cross Correlation maximization by Powell's method. The probe position and orientation, given by a mechanical arm holding the probe, are used for registration initialization. For 3D data sets of 10 patients acquired under different probe pressures, the resulting TRE is $3.18 \pm 1.6 \mathrm{~mm}$. More recently, De Silva et al. [6] proposes an enhanced algorithm whose optimization is based on learned prostate motion characteristics. After incorporating the learned initialization positions and directions in Powell's method, they obtain a registration error of $2.33 \pm 1.09 \mathrm{~mm}$ with $\sim 3 \mathrm{~s}$ mean execution time per registration. Gillies et al. [7] optimize the 2D-3D registration algorithm presented in [5] and obtain a computation times of $31 \mathrm{~ms}$ per registration in phantom experiments.

Khallaghi et al. [8] also proposed a slice-to-volume registration method for freehand prostate biopsies. It includes a trajectory-based rigid registration and a non-rigid model-based registration. The latter takes the prostate deformation into account by using a Finite Elements Model (FEM). Both stages use sum of square differences to determine image similarity. The 2D US probe is tracked using a magnetic system. The accuracy after non-rigid registration is $4.63 \pm 1.05$ $\mathrm{mm}$. The computation time is about $30 \mathrm{~s}$ (resp. $10 \mathrm{~min}$ ) for rigid and (resp. non-rigid) registration, which is not compatible with real-time navigation.

Our objective is to add real-time navigation abilities in the previously developed organ-based approach without the need for a complex localization device $^{1}$. We propose the following approach (Fig. 2): similarly to [4], a) a pre-operative 3D-3D MRI to US non-rigid registration may be performed at the beginning of the procedure in order to map the potential targets in a reference 3D US image; and $b$ ) a 3D-3D US to US non-rigid registration is also performed at each biopsy site in order to obtain an accurate localization

1 Cumbersome equipment such as an articulated arm to hold the probe or constraining equipment such as a magnetic localizer that can be perturbed by metallic objects. 


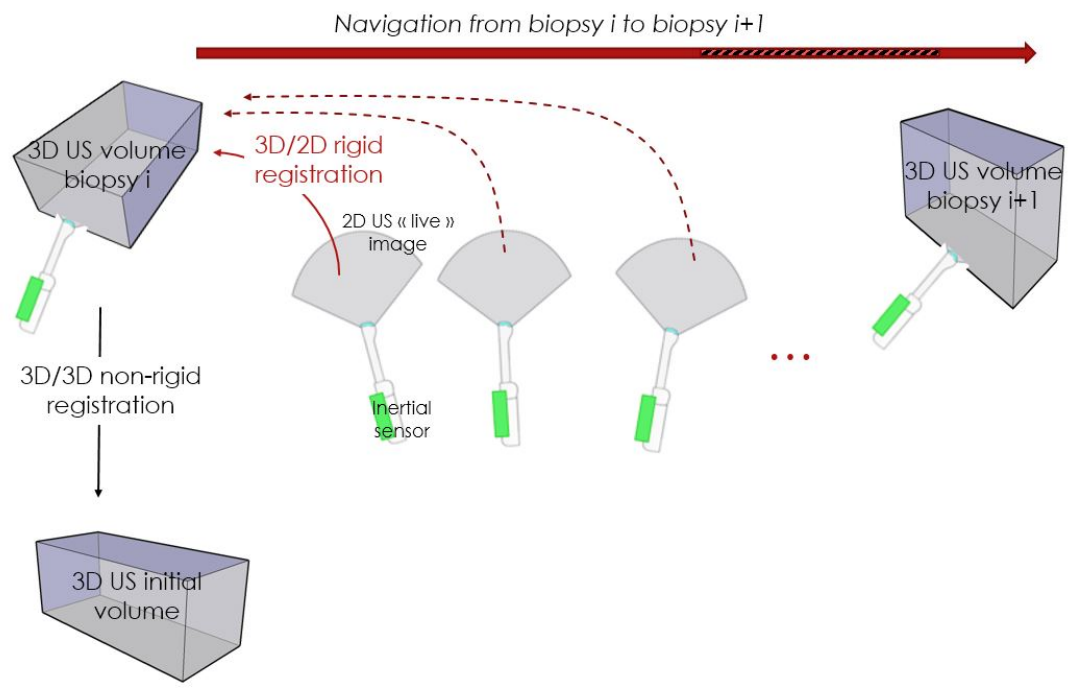

Fig. 2 The global approach

of the biopsy with respect to the reference image; in addition $c$ ), "live" 2D US images are continuously acquired and registered to the 3D US volume acquired at the previous biopsy site in order to provide a continuous guidance for the clinician between two biopsy site. To improve the slice-to-volume registration robustness, an inertial sensor is attached to the probe. This paper describes the hybrid method that rigidly and rapidly registers a live $2 \mathrm{D}$ image to a $3 \mathrm{D}$ volume.

The method introduces a variant of the Iterative Closest Point (ICP) algorithm using a combination of an euclidean distance of feature positions and a similarity distance of feature descriptors. The main steps of our algorithm are (see Fig. 3) : (1) feature extraction from live 2D and reference 3D ultrasound images, (2) feature description using pixel-based and voxel-based information, and (3) matching of nearby 2D-3D features in order to compute the spatial transformation between images. As many choices are possible for these three steps, we first estimated the sensitivity of the method to different parameter settings using an analysis of variance. Then, based on a selected set of parameter values, the performance of our method was evaluated in terms of accuracy and computation time on a large patient data set (160 volumes from 20 anonymized patients) randomly selected from routine biopsy performed in two different hospitals by 6 operators. Computed transformations were compared to ground truth transformations obtained using manually identified anatomical fiducials. Section 2 describes the registration method. Section 3 presents the validation approach, the conducted experiments and reports the obtained results. Section 4 includes a discussion and an outlook on future work. 


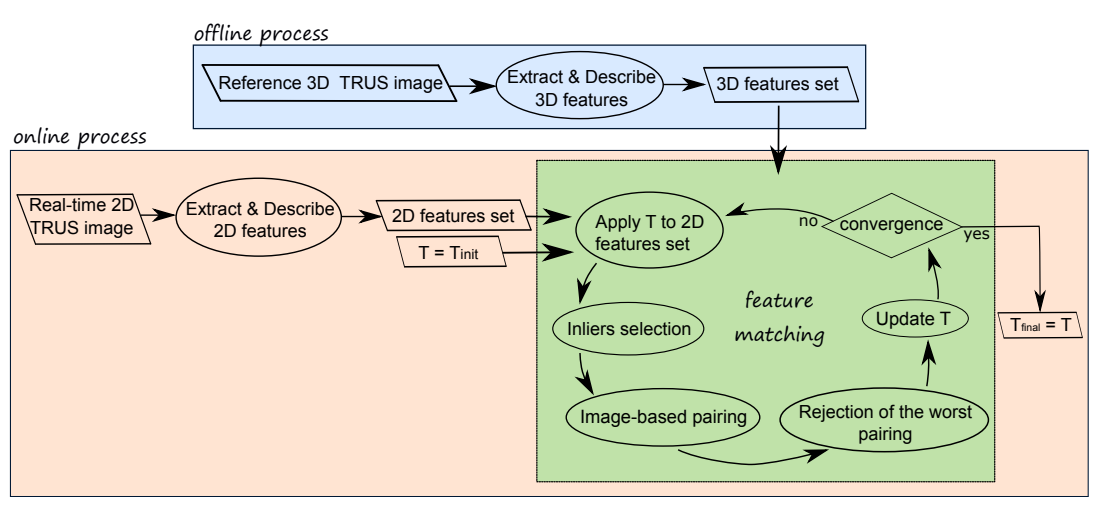

Fig. 3 Workflow of our algorithm
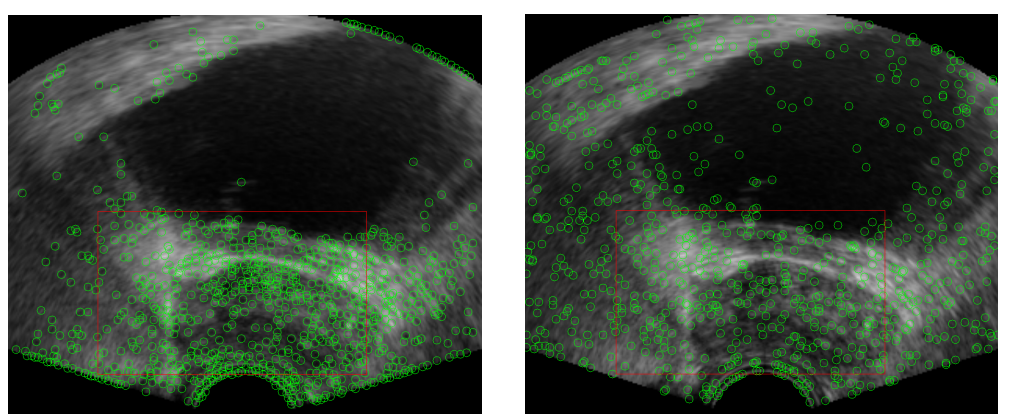

Fig. 4 Feature extraction using the Harris method (on the left) and the multi-scale method (on the right).

\section{Methods}

\subsection{Feature Extraction}

The first step of our method consists in automatically extracting the most significant interest points from the $2 \mathrm{D}$ live and $3 \mathrm{D}$ reference US images. These interest points are image salient points. 3D features are extracted only once, just before the biopsy starts, while the $2 \mathrm{D}$ features have to be extracted on each new live $2 \mathrm{D}$ image. The features are extracted on the whole images to avoid the need for prostate segmentation. This is especially important when considering the use of $2 \mathrm{D}$ live images and to permit registration of images where the prostate is only partly visible. We compared two different feature extraction methods (see Fig. 4) to detect significant intensity changes: the first one, also called the Harris method, detects rotation-invariant features, the second one, also called the multilevel method, detects scale and rotation-invariant features.

For rotation-invariant features, we use the Harris corner detection [12] based on Hessian matrix approximation. In order to reduce the computational cost, only the $N_{H}^{2 D}$ features with the highest corner response values are kept. 


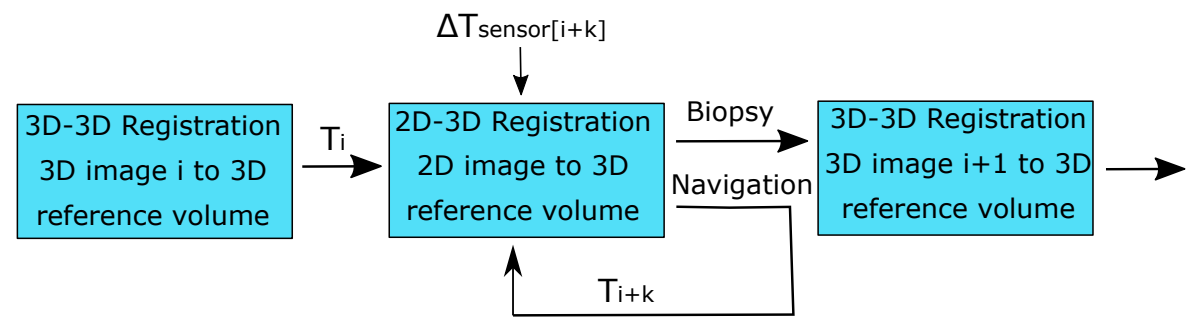

Fig. 5 Iterative loop of 2D-3D registrations performed between two 3D-3D registrations.

The same principles are applied to extract $N_{H}^{3 D}$ features in the $3 \mathrm{D}$ images. To achieve scale-invariance, a multilevel method is used to detect features using a multilevel image pyramid [10]. The idea consists in detecting key points identified for different image scales. Salient points are then extracted by locating local extrema in series of difference of Gaussian images for different sampling. The Gaussian distribution std $\operatorname{dev} \sigma_{2 D}$ and $\sigma_{3 D}$ controls the resulting number of features.

\subsection{Feature Matching}

The second step of our method consists in computing the best rigid transformation between the set of the $2 \mathrm{D}$ features $\mathbf{F}_{2 D}$ and the set of $3 \mathrm{D}$ features $\mathbf{F}_{3 D}$. This transformation is computed by a variant of ICP which works as follows.

\subsubsection{Initial Transformation.}

Firstly, the algorithm is initialized with a rough approximation $T_{i}$ transform given by a $3 \mathrm{D}-3 \mathrm{D}$ registration. A relative transform $\Delta T_{\text {sensor }[i+k]}$ estimating the probe displacement since the previous 3D-3D registration $T_{i+k}$ is computed and given for instance by an inertial sensor fixed on the US probe (see Fig. 5). This transformation initializes the process and each $2 \mathrm{D}$ feature can be initially positioned in the $3 \mathrm{D}$ image space.

\subsubsection{Geometry-Based Selection.}

Using this current position, candidates in $\mathbf{F}_{2 D}$, named inliers, are selected by considering their closest geometric neighbors in $\mathbf{F}_{3 D}$. To obtain an efficient selection, we use a $k d$-tree. Any $2 \mathrm{D}$ feature that has no $3 \mathrm{D}$ feature inside a neighborhood of radius $d_{\varepsilon}$ will not be considered in the next steps. This step is crucial for outliers elimination when only a part of $\mathbf{F}_{2 D}$ overlaps $\mathbf{F}_{3 D}$. This selection of a subset of possible matches prioritizes best candidates and reduces computational cost. Then, for each preselected feature $f_{2 D} \in \mathbf{F}_{2 D}$, the $n_{n}$ geometrically closest $3 \mathrm{D}$ feature neighbors are finally selected in $\mathbf{F}_{3 D}$. 


\subsubsection{Image-Based Pairing.}

The next stage of the ICP algorithm is to associate to each preselected 2D feature a single 3D feature among potential candidates. For each selected 2D and $3 \mathrm{D}$ features, we compute the image intensity $\mathbf{I}$, the mean intensity $\overline{\mathbf{I}}$, the intensity histogram $\mathbf{H}$ and the mean histogram $\overline{\mathbf{H}}=\frac{1}{N} \sum_{\Omega} \mathbf{H}(\Omega)$ for a given neighborhood $\Omega$, where $\mathrm{N}$ is the total number of histogram bins and possible range of the pixel value. These values are noted using an index of 1 and 2 for $2 \mathrm{D}$ feature and $3 \mathrm{D}$ feature respectively. We then define a similarity distance $\mathbf{d}\left(f_{2 D}, f_{3 D}\right)$ that combines three terms based on local intensity of the features:

$$
\mathbf{d}\left(f_{2 D}, f_{3 D}\right)=\alpha_{1} d_{1}+\alpha_{2} d_{2}+\alpha_{3} d_{3},
$$

where $\sum_{i=1}^{3} \alpha_{i}=1 . d_{1}=\frac{\left\|\overline{\mathbf{I}}_{1}-\overline{\mathbf{I}}_{2}\right\|}{N}$ compares the mean intensity in the neighborhood of the features.

$d_{2}$ uses the Bhattacharyya distance which quantifies the similarity of two normalized histograms: $d_{2}=\sqrt{1-\frac{1}{N \sqrt{\overline{\mathbf{H}}_{1} \overline{\mathbf{H}}_{2}}} \sum_{I} \sqrt{\mathbf{H}_{1}(I) \mathbf{H}_{2}(I)}} \cdot d_{3}$ is the sum of squared differences: $\mathbf{d}_{3}=\frac{1}{N^{2}} \frac{1}{|\Omega|} \sum_{j \in \Omega}\left(\mathbf{I}_{1}(j)-\mathbf{I}_{2}(j)\right)^{2}$.

At the end of this step, pairs composed of one 2D feature and one 3D feature are created by selecting for each $2 \mathrm{D}$ feature $f_{2 D} \in \mathbf{F}_{2 D}$ the $3 \mathrm{D}$ feature $f_{3 D} \in \mathbf{F}_{3 D}$ that minimizes $\mathbf{d}\left(f_{2 D}, f_{3 D}\right)$.

\subsubsection{Rejection.}

The pairs with the biggest $\mathbf{d}\left(f_{2 D}, f_{3 D}\right)$ are then rejected in order to eliminate potentially erroneous pairings. $p_{r}$ defines the percentage of such pairs.

\subsubsection{Updating Transformation.}

The final step of the ICP algorithm computes the transformation $T$ which minimizes the geometric distance between the remaining pairs using the Arun algorithm for direct least-square error minimization [11]. In order to give higher importance to the features located inside the prostate, pairs with a $3 \mathrm{D}$ feature inside the pre-segmented prostate are weighted with $w_{p}$. This partly overcomes the limits of rigid registration on the whole image. Other pairs have a default weight of 1 . The rationale is that information relative to the prostate could be more relevant for biopsy guidance using rigid registration.

\subsubsection{Stopping Criterion.}

The ICP algorithm convergence criterion is reached when a stable transformation $T$ is obtained. Let $R_{r e s}=R_{k-1}^{-1} * R_{k}$ be the the rotation difference and $t_{r e s}=t_{k}-t_{k-1}$ be the translation difference between the ICP iterations $k-1$ and $k$, we defined the convergence criterion as $\left\|R_{\text {res }}\right\|<10^{-3}$ and $\left\|t_{r e s}\right\|<0.05 \mathrm{~mm}$. If the convergence criterion is not satisfied, the algorithm starts again at the geometry-based selection step (see figure 3). A maximum 
number of iterations (150) found experimentally is used for stopping non converging registrations.

\section{Validation and Results}

In our previous feasibility study [9], the ability of the registration method to converge back to the original transform after applying a given perturbation was evaluated. To do this the patient data were used in a simplified way by artificially slicing a 3D volume of interest to generate 2D images, arbitrarily perturbing their positions, and finally registering them back to the 3D volume. Different feature extractors and parametrization were also explored. This approach provided a ground truth as the real position of the slice was exactly known. However, this did not take into account the complexity of the real case, where $2 \mathrm{D}$ live images have to be registered to a previously acquired $3 \mathrm{D}$ volume. The efficiency of the method could be extremely challenged by the presence of different noise patterns and potential deformation of the organ. This paper presents a more complex evaluation that includes a sensitivity analysis and a much more realistic $3 \mathrm{D} / 2 \mathrm{D}$ registration from routine patient data.

\subsection{Patient Data Set}

We randomly selected the validation database from a larger database of routinely acquired patient biopsy. The validation database contains 20 patient data sets for a total of 160 volumes acquired with two different versions of a commercial system (Urostation ${ }^{\circledR}$ and Trinity ${ }^{\circledR}$ from Koelis SAS). Volumes have been acquired using a 3D TRUS probe. They have been recorded during routine prostate biopsy procedures by six different operators from two different hospitals (Grenoble University Hospital Center and Paris University Hospital Center). Each anonymized patient data set is composed of a reference volume, acquired at the start of the procedure, and of 2 to 17 biopsy volumes, acquired for each performed biopsy sample. Live 2D US images are simulated by extracting the central image of the biopsy volumes. As the $3 \mathrm{D}$ volume position with respect to the gland depends on the biopsy site, the extracted central slice may show different part of the prostate in terms of size, shape, and position. As biopsy needles may be visible in these images (see figures 7.a and 8.a), a local mask is applied to discard $2 \mathrm{D}$ features at the needle location in the $2 \mathrm{D}$ image.

\subsection{Ground Truth}

For each biopsy volume, we identified from 4 to 6 anatomic fiducials within the prostate (visible micro-calcifications) also visible in the corresponding patient reference volume to create fiducial pairs. Volumes that did not contain enough 


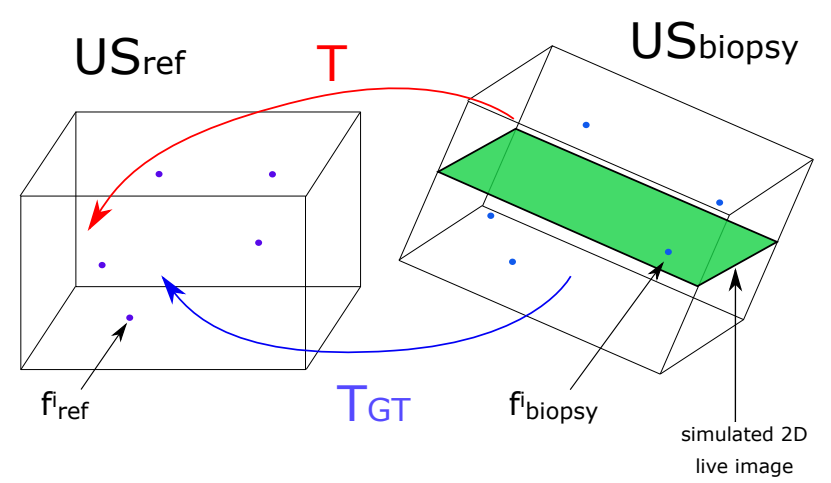

Fig. 6 In our experiment, the slice extracted from the biopsy volume is registered to the reference volume with the gold standard transform $T_{G T}$.

visible micro-calcifications were discarded from the validation database. These fiducial pairs were used to compute a ground truth transformation $T_{G T}$ using paired-point matching [11]. To estimate the manual landmarks segmentation error, we repeated the fiducial selection on 5 different patient volumes once every day for 5 days and obtained a variance of $0.38 \mathrm{~mm}$. The Fiducial Registration Error (FRE) between a given reference volume and a given biopsy volume is defined by $\operatorname{FRE}(\mathrm{mm})=\sqrt{\frac{\sum_{i=1}^{N_{f}}\left\|f_{r e f}^{i}-T_{G T}\left(f_{b i o s p y}^{i}\right)\right\|^{2}}{N_{f}}}$, where $f_{\text {ref }}^{i}$ and $f_{\text {biopsy }}^{i}$ are the $i^{\text {th }}$ manually paired fiducial positions and $N_{f}$ the number of fiducials. The mean \pm std FRE for the 160 volumes is $0.74 \pm 0.34 \mathrm{~mm}$. This relatively small FRE shows that using a rigid registration on actual clinical data-sets is a reasonable choice.

\subsection{Registration Under Simulated Rotation and Translation}

For each biopsy volume, artificial rotations and translations were generated from the initial $T_{G T}$ in order to simulate the TRUS probe or prostate movements. The ranges of the disturbance were chosen to simulate an acquisition noise on the probe position given by the inertial sensor. Experiment 1 consists in varying a single degree of freedom (DOF) of the rigid transformation: the range of the rotation DOF disturbance was $\left[-15^{\circ}, 15^{\circ}\right]$ (by steps of $1^{\circ}$ ) and the range of the translation DOF disturbance was $[-15 \mathrm{~mm}, 15 \mathrm{~mm}$ ] (by steps of $1 \mathrm{~mm}$ ). This resulted in 186 different initial transformations. Experiment 2 consists in randomly selecting 25 disturbances six DOF at the same time: the rotation range was $\left[-5^{\circ}, 5^{\circ}\right]$ and the translation range was $[-7.5 \mathrm{~mm}, 7.5 \mathrm{~mm}]$. Experiments 1 and 2 are repeated for each of the 160 biopsy volumes. 


\subsection{Parameter Sensitivity Analysis}

Among all possible parameter combinations, we selected a limited number of configurations for the parameter sensitivity study based on our previous work [9]. Experiment 1 was therefore performed in 20 configurations, corresponding to 420,360 registrations.

We focused on the feature extraction method, the number of features and inliers selection and studied the effect of:

- Feature extraction method: either the Harris method with $\left(N_{\mathrm{H}}^{2 \mathrm{D}}=300\right.$, $\left.N_{\mathrm{H}}^{3 \mathrm{D}}=20,000\right)$ or with $\left(N_{\mathrm{H}}^{2 \mathrm{D}}=350, N_{\mathrm{H}}^{3 \mathrm{D}}=25,000\right)$, or the multi-scale method with $\left(\sigma_{2 D}=0.6^{2}\right.$ and $\left.\sigma_{3 D}=0.5\right)$ or $\left(\sigma_{2 D}=0.5\right.$ and $\left.\sigma_{3 D}=0.35\right)$

- Inliers selection distance: $d_{\varepsilon}=1.5 \mathrm{~mm}, d_{\varepsilon}=2 \mathrm{~mm}$ or no outliers elimination,

- Similarity distance weights: either $\left(\alpha_{i}=\frac{1}{3}\right)$ or $\left(\alpha_{1}=\frac{1}{2} ; \alpha_{2}=\frac{1}{2} ; \alpha_{3}=0\right)$,

- Number of geometrically closed 3D features selected: $n_{n}=5$ or $n_{n}=10$,

- Rejection rate: $p_{r}=0 \%, p_{r}=10 \%$ or $p_{r}=20 \%$,

- Weight for the pair inside the prostate: $w_{p}=1.0, w_{p}=4.0$ or $w_{p}=10.0$,

- $\Omega$ size is fixed to 10 (based on previous experiments [9]).

We use a multivariate general linear model on a subset of volumes of the data set. Statistical analysis of results using $\mathrm{R}$ package showed some significant differences on mean TRE. For instance, choice of feature type, inliers selection and weight for the pair inside the prostate had a significant impact on the accuracy and the trends were homogeneous (TRE always increasing or decreasing depending on the choice). For the others, we chose parameters with an optimal ratio accuracy/computation time. The ANOVA studies showed that the best results were obtained with the multi-scale method, $\sigma_{2 D}=0.5$ and $\sigma_{3 D}=0.35, d_{\varepsilon}=2 \mathrm{~mm}, \alpha_{1,2,3}=\frac{1}{3}, n_{n}=5, p_{r}=0 \%$, and $w_{p}=4.0$. The remaining experimental studies were conducted using these values.

\subsection{Experimental Results}

\subsubsection{Registration Accuracy.}

The mean TRE using the anatomical fiducials were computed for experiments 1 and 2 before and after rigid registration, see Table 1 . We performed a Wilcoxon rank sum test which rejects the hypothesis that TREs for preregistration vs after registration belong to a distribution with equal medians. The improvements in mean TRE is statistically significant. Considering that clinically significant tumor are above $0.5 \mathrm{cc}$ (equivalent to a sphere of radius $5 \mathrm{~mm}$ )[13], TRE under $5 \mathrm{~mm}$ is clinically acceptable and relevant. $74.8 \%$ of overall TRE are under $5 \mathrm{~mm}$. Figure 7 provides an example of registration. Figure 9 plots registration accuracy for one patient with 17 biopsy datasets.

2 For the muti-scale method, the number of features is not preset but depends on image quality or echogenicity and is related to the selected size of the Gaussian. 

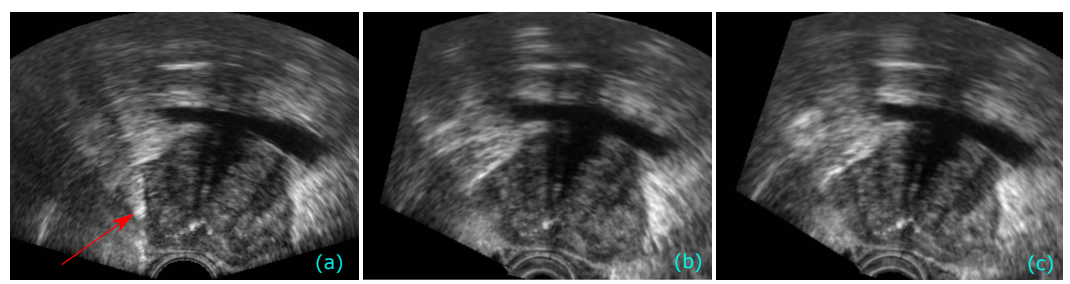

Fig. 7 Registration example. (a) 2D live image acquired during a biopsy (red arrow points to the needle). (b) Corresponding slice extracted from the reference volume after registration $(\mathrm{TRE}=1.48 \mathrm{~mm}$ - before registration $\mathrm{TRE}=9.31 \mathrm{~mm})$. (c) Slice extracted from the reference volume using Ground truth transformation $(\mathrm{FRE}=0.48 \mathrm{~mm})$.
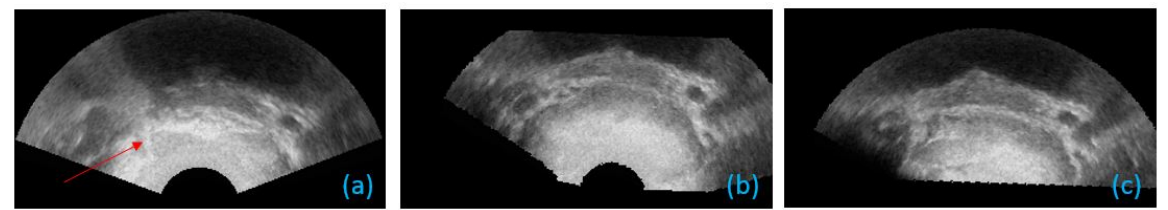

Fig. 8 Example of inaccurate registration. (a) 2D live image acquired during a biopsy (red arrow points to the needle). (b) Corresponding slice extracted from the reference volume after registration $(\mathrm{TRE}=9.14 \mathrm{~mm}$ - before registration $\mathrm{TRE}=15.02 \mathrm{~mm})$. (c) Slice extracted from the reference volume using Ground truth transformation ( $\mathrm{FRE}=1.37 \mathrm{~mm}$ ).

Table 1 Registration results

\begin{tabular}{|c|c|c|c|}
\hline & Number of registrations & TRE before registration & TRE after registration \\
\hline Experiment 1 & 29,760 & $5.63 \pm 4.12 \mathrm{~mm}$ & $3.91 \pm 3.22 \mathrm{~mm}$ \\
\hline Experiment 2 & 4,000 & $7.48 \pm 2.25 \mathrm{~mm}$ & $4.37 \pm 2.62 \mathrm{~mm}$ \\
\hline
\end{tabular}

It is important to underline that these results include all the registrations even non converging registrations which represents $2.8 \%$ of all registrations. The non-convergence sources of these are : (1) a lack of image overlap when the biopsy is performed close to organ extremities (apex, base, very lateral biopsy) - see for instance figure 8, (2) a very large prostate deformation or (3) a low quality of the images, limiting the number of extracted features.

\subsubsection{Computation Times.}

Our method is implemented in $\mathrm{C}++$ without any code optimization using the CamiTK [14] open source development framework. The code was executed on a Windows 7 machine with an Intel Core i7-3840QM. The computational complexity of the feature extraction is linear in the number of pixels/voxels. The matching is $\mathcal{O}(n \log n)$ where $n$ is the number of $2 \mathrm{D}$ features. Before starting the procedure, the $3 \mathrm{D}$ features are extracted in about $8 \mathrm{~s}$. The computation time of the $2 \mathrm{D}$ features extraction is in the order of $1.9 \mathrm{~s}$ for 1,000 features. For the multi-scale method, the obtained number of features which depends directly on the value of $\sigma$ (cf. section 3.4), varied from around 600 to around 800. The mean number of iterations of the ICP algorithm is $45.9 \pm 29.4$. The 


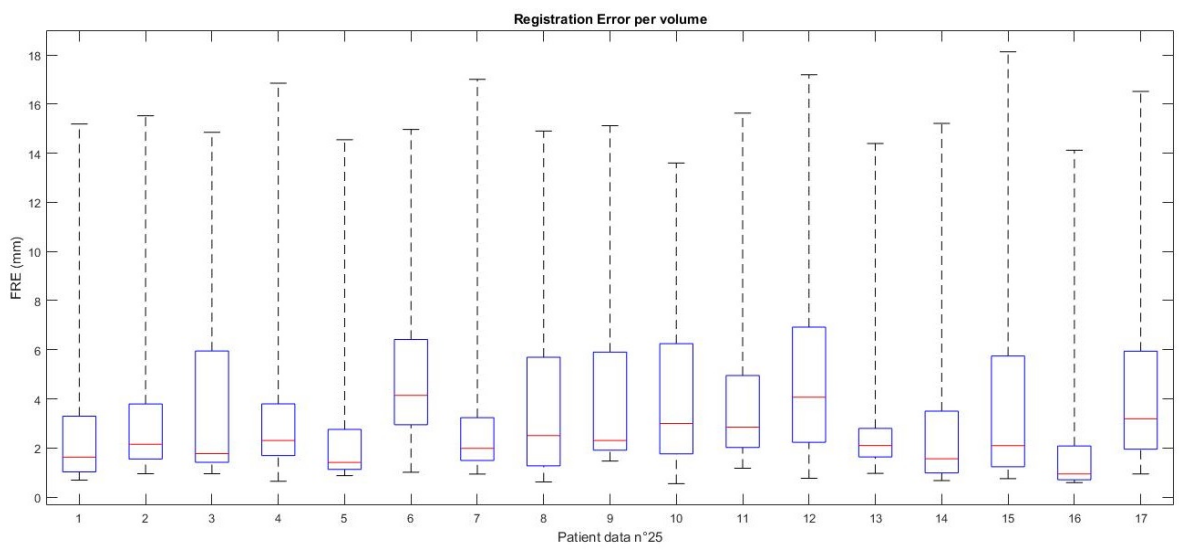

Fig. 9 Registration performance for one patient (experiment 1). The box plot shows results for the 186 registrations per biopsy volume

mean computation time of the ICP algorithm is $1.8 \mathrm{~s}$ and ranges from $40 \mathrm{~ms}$ to $6000 \mathrm{~ms}$. We classically observed that the convergence is faster when the registration transformation is closer to the solution.

\section{Discussion and conclusion}

We proposed a 2D-3D ultrasound registration for navigated prostate biopsy and its evaluation on a significant patient data set recorded in routine uncontrolled conditions. For a total of 160 volumes acquired on 20 randomly selected patients, 35,760 registrations were run exploring possible initializations. The accuracy was measured on anatomical fiducials (4 to 6 per volume). The results (cf. table 1) have to be discussed with respect to related work. In [5], for a total of 16 registrations involving 16 live $2 \mathrm{D}$ images for 8 patients, the TRE was $1.87 \pm 0.81 \mathrm{~mm}(4.75 \pm 2.62 \mathrm{~mm}$ before registration). This was evaluated on fiducials extracted in 2D slices and 3D data (3 or 4 per image). The initialization came from the position of the probe held by a robotic arm. In a second dedicated set-up, three $3 \mathrm{D}$ volumes of 10 patients were recorded with 3 levels of probe pressure; slices were simulated in 2 of the 3 volumes and registered to the third one. For the 20 registrations, the TRE measured on $3 \mathrm{D}$ fiducials was $3.18+/-1.6 \mathrm{~mm}$ ( $6.89 \pm 4.1 \mathrm{~mm}$ before registration). In [8], 115 registrations for 10 volumes and 115 live 2D images were executed based on the trajectory and orientation of the probe from magnetic tracking. Measured on fiducials extracted on 2D and 3D data (about 6 per image), the TRE after non rigid registration was $4.63 \pm 1.05 \mathrm{~mm}(6.31 \pm 1.86 \mathrm{~mm}$ before registration). The accuracy we obtained is slightly lower or similar to the one reported in the literature but it must be kept in mind that it corresponds to experiments on a much larger data set obtained from routine recorded data. Also, we com- 
puted gold standard transforms based on fiducials on 3D volumes, whereas in [8] and [5], the fiducials were identified on 2D slices. Our gold standard transforms computed with $3 \mathrm{D}$ fiducials are therefore probably more precise. The testing also explores in a more systematic way the basin of attraction of our method. This shows that our method is rather robust in spite of inaccurate initialization. Let us stress the fact that in clinics, such inaccurate initialization may happen very often due to prostate or patient uncontrolled motions. In [8], the computation time is about $30 \mathrm{~s}$ (resp. $10 \mathrm{~min}$ ) for rigid and (resp. FEM-based) registration which is not compatible with real-time navigation. [5] reports mean running time of $1.1 \mathrm{~s}$ for the experiments listed above with a GPU implementation of their method. Considering the potential of parallelization of most of our method steps, we are optimistic about our ability to significantly decrease the computational cost of our method also using GPU to reach similar performances.

The proposed framework is intended to be incorporated in a navigation process with repeated registration during probe motion from one biopsy to the following. [5] reports the improvement of the registration accuracy (from 1.63 to $1.81 \mathrm{~mm}$ in average) on the same database of 16 images for 8 patients by repeating the registration every second. Repeated registration also results in better initialization and improved convergence. This could also allow us to decrease $n_{n}$ to improve the performance. Moreover, a GPU implementation would give us the possibility to test more parameters values.

The next step consists in evaluating our method on real conditions using inertial sensor data and images during a live session.

Ethical statement: All procedures performed in studies involving human participants were in accordance with the ethical standards of the institutional and/or national research committee and with the 1964 Helsinki declaration and its later amendments or comparable ethical standards.

Conflict of Interest: The authors declare that they have no conflict of interest.

\section{References}

1. V. Iremashvili, L. Pelaez, M. Jorda, M. Manoharan, M. Arianayagam, D. Rosenberg and M. Soloway. Prostate Sampling by 12-Core Biopsy: Comparison of the Biopsy Results With Tumor Location in Prostatectomy Specimens Urology, 79(1):37-42,2012.

2. S. Xu, J. Kruecker, B. Turkbey, N. Glossop, A. Singh, P. Choyke, P. Pinto, and B. Wood Real-time MRI-TRUS fusion for guidance of targeted prostate biopsies

3. J. Bax, D. Cool, L. Gardi, K. Knight, D. Smith, J. Montreuil, S. Sherebrin, C. Romagnoli, and A. Fenster. Mechanically assisted 3d ultrasound guided prostate biopsy system. Medical Physics, 35(12):5397-5410, December 2008.

4. Baumann, M. and Mozer P. and Daanen V. and Troccaz J. Prostate biopsy tracking with deformation estimation. Medical Image Analysis 2011, 16, 562-576.

5. T. De Silva, A. Fenster, D. Cool, L. Gardi, C. Romagnoli, J. Samarabandu, and A. Ward. 2d-3d rigid registration to compensate for prostate motion during 3d TRUS-guided biopsy. Medical Physics, 40(2):022904, 2013.

6. De Silva, Tharindu and Cool, Derek and Yuan, Jing and Romagnoli, Cesare and Samarabandu, Jagath and Fenster, Aaron and Ward, Aaron Robust 2D-3D registration optimization for motion compensation during 3D TRUS-guided biopsy using learned prostate motion data IEEE Transactions on Medical Imaging, 2017. 
7. Gillies, Derek J and Gardi, Lori and Zhao, Ren and Fenster, Aaron Optimization of realtime rigid registration motion compensation for prostate biopsies using $2 \mathrm{D} / 3 \mathrm{D}$ ultrasound SPIE Medical Imaging, 101351F-101351F, 2017.

8. S. Khallaghi, C. Sanchez, S. Nouranian, S. Sojoudi, S. Chang, H. Abdi, L. Machan A. Harris, P. Black, M. Gleave, L. Goldenberg, S. Fels, and P. Abolmaesumi. A 2d3d Registration Framework for Freehand TRUS-Guided Prostate Biopsy. MICCAI 2015, 9350, 272-279.

9. S-Y. Selmi, E. Promayon, J. Troccaz. 3D-2D ultrasound feature-based registration for navigated prostate biopsy: A feasibility study. 38th Annu. Int. Conf. IEEE Eng. Med. Biol. Soc., 2016, pp. 41094112

10. D. Lowe. Distinctive Image Features from Scale-Invariant Keypoints, International Journal of Computer Vision, 60(2):91-100,2004.

11. K.S. Arun, T.S. Huang, and S.D. Blostein. Least-Squares Fitting of Two 3-D Point Sets. IEEE Transactions on Pattern Analysis and Machine Intelligence, PAMI-9(5):698700,1987 .

12. C. Harris and M. Stephens. A combined corner and edge detector. pages $147-151,1988$.

13. Cornud, F., L. Brolis, N. Barry Delongchamps, D. Portalez, B. Malavaud, R. RenardPenna, and P. Mozer. TRUS-MRI Image Registration: A Paradigm Shift in the Diagnosis of Significant Prostate Cancer. Abdominal Imaging 38, no. 6, 2013

14. C. Fouard, A. Deram, Y. Keraval, E. Promayon. CamiTK: a Modular Framework Integrating Visualization, Image Processing and Biomechanical Modeling. In Soft Tissue Biomechanical Modeling for Computer Assisted Surgery, Y. Payan (ed.), pp. 323-354, 2012 